\title{
Publisher Correction: N-terminal syndecan-2 domain selectively enhances 6-O heparan sulfate chains sulfation and promotes VEGFA $165^{-}$ dependent neovascularization
}

Federico Corti ${ }^{1}{ }^{1}$, Yingdi Wang ${ }^{1}$, John M. Rhodes ${ }^{1}$, Deepak Atri ${ }^{1}$, Stephanie Archer-Hartmann ${ }^{2}$, Jiasheng Zhang ${ }^{1}$, Zhen W. Zhuang ${ }^{1}$, Dongying Chen ${ }^{1}$, Tianyun Wang ${ }^{1}$, Zhirui Wang ${ }^{2}$, Parastoo Azadi ${ }^{2}$ \& Michael Simons (1) ${ }^{1,3}$

Correction to: Nature Communications https://doi.org/10.1038/s41467-019-09605-z, published online 05 April 2019.

The original version of this Article contained errors in Figs. 1, 3 and 4. In panels b and d of Fig. 1, the labels 'Sdc4 ${ }^{-/-}$' were inadvertently

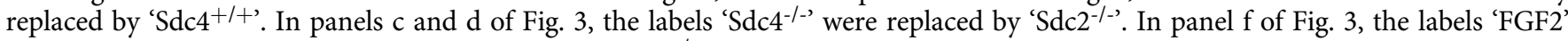
were replaced by 'VEGFA 165 '. In panel e of Fig. 6 , a 'Sdc2 ${ }^{-/-c}$ label was inadvertently included. This has now been corrected in the PDF and HTML versions of the Article.

Published online: 07 May 2019

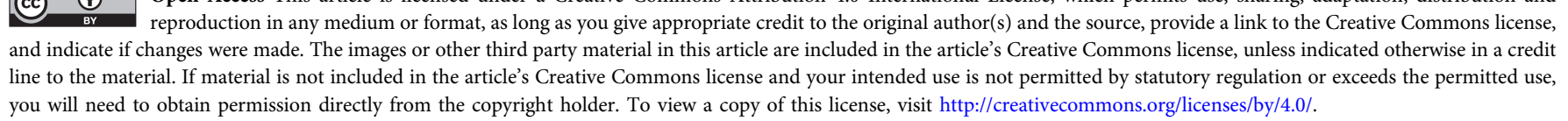

(C) The Author(s) 2019

\footnotetext{
${ }^{1}$ Yale Cardiovascular Research Center, Section of Cardiovascular Medicine, Department of Internal Medicine, Yale University School of Medicine, 300 George Street, New Haven, CT 06511, USA. ${ }^{2}$ Complex Carbohydrate Research Center, The University of Georgia, 315 Riverbend Road, Athens, GA 30602 , USA. ${ }^{3}$ Department of Cell Biology, Yale University School of Medicine, New Haven, CT 06520, USA. These authors contributed equally: Federico Corti, Yingdi Wang. Correspondence and requests for materials should be addressed to M.S. (email: michael.simons@yale.edu)
} 